\title{
OPRESIÓN MEDIÁTICA DE LOS SECTORES EMPOBRECIDOS EN EL PUERTO RICO MODERNO
}

\author{
Yalitza Navedo Román
}

\section{Resumen}

El presente trabajo plasma un marco teórico conceptual con el objetivo de entender el fenómeno de la invisibilización-hipervisibilización como una opresión mediática que afecta a sectores empobrecidos y, a la vez, trazar una ruta alterna para lograr dar visibilidad positiva a estos segmentos poblacionales. Quiñones Rosado (2007), Quijano (2000), Dussel (2007), Hardiman y Jackson (1997), Bronfenbrenner (1987) y Melano (2001), son algunos de los autores trabajados en este marco. [Palabras clave: invisibilizaciónhipervisibilización mediática, sectores empobrecidos en los medios, medios de comunicación masiva y Trabajo Social].

\section{Abstract}

The present work reflects a conceptual theoretical framework in order to understand the phenomenon of the invisibilization-hipervisibilization as an oppression media affecting impoverished sectors and, at the same time, stroking an alternate path to get a positive visibility to these population segments. Quiñones Rosado (2007), Quijano (2000), Dussel (2007), Hardiman y Jackson (1997), Bronfenbrenner (1987) y Melano (2001), are some of the authors worked within this framework. [Keywords: media invisibility-hipervisibility, the poor in the media, mass media and Social Work].

\section{Introducción}

La invisibilización ${ }^{2}$ de los sectores empobrecidos en los medios es un tema que ha ocupado discusiones académicas y profesionales desde los años sesenta. Por ejemplo, Mujica (1969) en un discurso en la Cámara de Diputados de Venezuela y bajo el tema Se ha impuesto el silencio a los pobres alerta sobre el asunto. Explica que este silencio se ha impuesto mediante la concentración en pocas manos de los medios de comunicación colectiva.

Esta misma preocupación fue denunciada hace unos años por Rafael Correa, presidente de Ecuador, en la apertura de la Comunidad de Estados Latinoamericanos y Caribeños 2011 (CELAC). Este mandatario mencionó que es un grave problema el poder fáctico de los medios de comunicación masiva privados porque atenta contra la verdadera libertad de expresión. Esencialmente, la problemática estriba en que la información se ha convertido en negocio. Explica que la libertad de expresión ha sido reducida a la supuesta libertad de prensa que sin ética, ni profesionalismo, ni los debidos controles sociales, ha sido reducida, a su vez, en una libertad de empresa que busca lucro o poder (Correa, CELAC, 8 de diciembre de 2011). Es decir, de acuerdo con el mandatario, los grandes empresarios dueños de los medios, establecen - conforme a su voluntad - lo que es la libertad de expresión. Una libertad de expresión caracterizada por la invisibilización de grandes sectores poblacionales.

De otra parte, son múltiples las quejas de los sectores que han sufrido la invisibilización de su gesta positiva e hipervisibilización de su gesta negativa en los medios de comunicación del país. De hecho, en una entrevista a profundidad que se le realizó a un líder comunitario de la Comunidad Especial Los Filtros en Guaynabo, se puede observar esta representación en los medios, cuando expresa:

Ejemplo, si en una comunidad se salvan diez, tal vez la prensa no va a resaltar que se

\footnotetext{
Piedras.

${ }^{1}$ Maestra en Comunicaciones. Estudiante del Programa Doctoral de Trabajo Social, Universidad de Puerto Rico, Recinto de Río

${ }^{2}$ Término utilizado para describir el proceso en que se silencian voces.
} 
salvaron diez, pero si matan diez va toda la prensa porque hay que resaltar que hubo una masacre, mataron diez. Entonces nosotros por esa parte, por lo menos en el caso de los grupos con los que yo trabajo, lo hemos absorbido y lo hemos visto como una cosa, pues, como un negocio... (Comunicación personal, 19 de enero de 2013)

Concurren con el líder comunitario Sepúlveda, García De Jesús, Escalante Rengifo y Toro Adorno (2002), quienes puntualizan: "En esa combinación de silencios y visibilidades se hipertrofia la violencia como cotidianidad irrefrenable en la ciudad y se invisibilizan las prácticas de ciudadanía y los comportamientos de paz". (pp. xiii-xiv)Este tipo de cobertura mediática desbalanceada nos hace postular que la invisibilización-hipervisibilización de los sectores empobrecidos en los medios de comunicación masiva podría estar fungiendo como una especie de opresión sistémica hacia los sectores en desventaja social y económica. En la siguiente sección abordamos el tema de la opresión para movernos más adelante a lo que sería, desde nuestra perspectiva, la opresión mediática.

\section{La opresión}

Quiñones Rosado (2007) se adentra, teniendo como contexto conceptual aspectos de la Wilber's integral theory y el Four Worlds'medicine wheel model's en la temática de cómo las fuerzas sistémicas en la sociedad subyugan a la gente todos los días. Explica que el sistema de poder diferenciado privilegia ciertos grupos sobre otros. Esta situación limita el bienestar de la humanidad porque se desarrolla la opresión de ciertos sectores o grupos sociales. Este incorpora un análisis de las fuerzas sociales, culturales, económicas y políticas que ejercen opresión estructural e impactan a las personas subordinadas por simplemente ser miembros de cierto grupo o sector. Más específicamente, Quiñones Rosado (2007) analiza la dinámica del racismo, etnocentrismo, colonialismo, clasismo, sexismo y heterosexismo, y sus efectos de internalización de esas diferentes formas de opresión. Este autor analiza esta situación de opresión dentro del contexto de Estados Unidos y su colonia, Puerto Rico. También analiza la situación opresiva de la comunidad latino y afroamericana en los Estados Unidos.

A raíz de esta opresión institucional, se manifiesta una inferioridad internalizada. Para lograr superar esta situación, el autor propone moverse de la perspectiva fragmentada a la visión integral de la realidad social. Explica que tanto en Puerto Rico como en los Estados Unidos, ha observado que los movimientos sociales trabajan de forma fragmentada lo que dificulta que se logren cambios en la estructura social opresiva. El autor invita a trabajar de forma integral y no fragmentadamente para lograr un entendimiento profundo de las problemáticas sociales y concretizar cambios significativos.

Quiñones Rosado (2007) - aunque enfoca su análisis en las relaciones de poder como la Teoría de la Complejidad de Edgar Morin, y no en los equilibrios naturales como la Teoría de Sistemadefinitivamente, concurre con la noción sistémica cuando resalta la importancia de observar los fenómenos a nivel sistémico u holístico (como él lo describe). La Teoría de Sistema, trabajada por Hull y Mather (2006), Anderson (1994), Greene (1991), Valle-Ferrer (1990), entre otros teóricos y académicos de trabajo social, explica que la familia, al igual que los individuos, las comunidades y las sociedades se componen de partes interdependientes; que si se afecta una parte, se afectan las otras, y como resultado, todo el sistema. La noción sistémica define un sistema como un conjunto de elementos relacionados entre sí y en continua interdependencia con el contexto o entorno que los rodea. Por su parte, en el trabajo de Quiñones Rosado (2007), se destaca la interdependencia entre lo individual y lo colectivo, es decir, la coexistencia interdependiente de todos los segmentos de la sociedad individuos, comunidad e instituciones - todo esto cruzado por la cuestión política, cultural, económica y social. Este razonamiento de Quiñones Rosado (2007) converge también con la teoría ecológica de Urie Bronfenbrenner que ayuda a comprender la relevante influencia de los ambientes en el desarrollo y formación del ser humano. Bronfenbrenner (1987) argumenta que la capacidad de formación de un sistema depende de la existencia de las interconexiones entre ese sistema y otros. Todos los niveles del modelo ecológico propuesto dependen unos de otros y, por tanto, se requiere de una participación conjunta de los diferentes contextos y de una comunicación entre ellos. Quiñones Rosado (2007) articula

${ }^{3}$ La Wilber's integral theory es un mapa comprensivo de las capaciddes humanas y el Four World's medicine wheel model presenta unesquema de pensamiento integral para guiar la acción a través de catro círculos interrelacionados: mental, emocional, físico y espiritual (Quiñones Rosado, 2007, pp.27 y 34). 
este asunto como "una mirada holística que plantea la coexistencia interdependiente entre la dimensión individual y colectiva" (p. 52). Sin embargo, su efoque - a diferencia de las nociones sistémicas y ecológicas - va más dirigido a analizar las relaciones de poder que se dan entre las dimensiones individual y colectiva.

En el modelo de Quiñones Rosado la esfera que contiene las estructuras política, cultural, económica y social es la llamada dimensión colectiva. Las estructuras mental, espiritual, física y emocional recogen la dimensión personal. Lo importante es saber que existe una interdependencia entre ambas esferas En el círculo pequeño del centro de estas dos dimensiones el autor ubica la conciencia y voluntad. Y precisamente, hay que tener conciencia de cómo se interrelacionan todas las esferas para mejorar la vida en sociedad. No se puede lograr el bien común, si no se impactan todas las estructuras en ambas dimensiones. Esta aseveración también aplica a la inversa, si se afecta alguna de las estructuras, se afectarán todas las estructuras interconectadas.

En fin, en Consciousness-in-Action (2007) el Dr. Raúl Quiñones Rosado sostiene que la opresión es un sistema de sistemas y trae a colación la siguiente fórmula:

$$
\text { PREJUICIO + PODER = OPRESIÓN }
$$

Es decir, desde la perspectiva integral, "opresión es un sistema de sistemas de fuerzas dinámicas sociológicas y sicológicas que afectan negativamente todos los aspectos, niveles y dimensiones de la esfera de la actividad humana" (Quiñones Rosado, 2007, p. 77, traducción de la autora). Este autor menciona a Hardiman y Jackson (1997), quienes especifican cuatro elementos clave para que exista la opresión:

- El agente opresor tiene poder y autoridad para definir, nombrar y determinar qué es "real", "normal" o "correcto" en la sociedad.

- Trato diferencial y desigual institucionalizado y sistematizado hacia los miembros del grupo oprimido. Esto puede ocurrir de forma consciente o inconsciente.

- La condición de opresión es internalizada sicológicamente por el grupo oprimido a través de la socialización resultando en su asimilación de la ideología y sistema social opresor.

- La cultura opresora es difundida por toda la sociedad mientras que la historia y cultura del grupo oprimido es pseudo-representada, descontinuada o erradicada (Quiñones Rosado, 2007, p. 77, traducción de la autora).

Estos elementos son clave para que exista opresión y, definitivamente, la relación existente entre los medios y los sectores empobrecidos apunta a que se está concretizando una opresión mediática que redunda en la invisibilización-hipervisibilización de los sectores marginados. En la siguiente parte se trabaja este asunto.

\section{Opresión mediática}

En las sociedades actuales los medios de difusión masiva juegan un papel vital en la vida individual, familiar y colectiva. Mitchel Stephens (2003) sostiene que estos medios influyen en la vida cotidiana ya que el intercambio de información se convierte en la base a partir de la cual se crea comunidad y se conforman las relaciones humanas. Sin embargo, a pesar de la importancia de los medios en la construcción de las sociedades modernas, existen sectores que no tienen acceso equitativo en los medios de difusión masiva. Rosanna Reguillo (2000) acuña el concepto de mapa de silencios. Esta autora explica que la situación de silencios (invisibilización) en los medios muestra las relaciones entre el poder, los medios de comunicación y los ciudadanos. Desde otra perspectiva, Mujica (1969) ya lo había planteado alegando que se les ha impuesto el silencio a los pobres. Cuestión que NoelleNeumann (1984), trabajando el tema de la opinión pública, denomina como espiral del silencio. Se trata 
de una situación de desbalance en la cobertura mediática en la que, generalmente, se invisibiliza la gesta positiva de los sectores empobrecidos.

Como se mencionó, Hardiman y Jackson (1997) especifican cuatro elementos clave para que exista la opresión: 1) El agente opresor tiene poder y autoridad para definir, nombrar y determinar que es "real", "normal" o "correcto" en la sociedad (los medios tienen este poder y autoridad); 2) Trato diferencial y desigual institucionalizado y sistematizado hacia los miembros del grupo oprimido (la invisibilizaciónhipervisibilización de los sectores empobrecidos es una muestra de esto); 3) La condición de opresión es internalizada sicológicamente por el grupo oprimido a través de la socialización resultando en su asimilación de la ideología y sistema social opresor (Quiñones Rosado puntualiza que esto lo ha observado en su trabajo con diversas comunidades); y 4) La cultura opresora es difundida por toda la sociedad mientras que la historia y cultura del grupo oprimido es pseudo-representada, descontinuada o erradicada (en el caso de los sectores empobrecidos en Puerto Rico se proyecta un retrato negativo que no representa la totalidad de la comunidad).

Si se examina con detenimiento, esto es precisamente lo que acontece a diario con los sectores empobrecidos en los medios de comunicación masiva de Puerto Rico. La invisibilización de los eventos positivos combinada con la hipervisibilización de los eventos negativos es un tipo de opresión que ha llevado a pseudorepresentar a los sectores desventajados del país. De hecho, representantes de los medios en Puerto Rico aceptaron en Tendiendo Puentes: Estudio para fortalecer la relación entre los medios de comunicación y las comunidades en Puerto Rico (2004) que los medios de comunicación masiva son empresas que responden a intereses económicos, y por tanto, privilegia cierto tipo de información sobre otra. No está demás mencionar que las informaciones de los sectores empobrecidos, definitivamente, no caen dentro de esta categoría de información privilegiada.

Quiñones Rosado (2007), al presentar el impacto de las opresiones en la identidad establece que esta emerge durante y como resultado del proceso de socialización, desarrollado por un contexto dinámico de interacción entre las personas en la dimensión colectiva. Este proceso, añade el autor, involucra la formación de la identidad impactando la imagen propia, el autoconcepto, la autoestima y el amor propio. Simultáneamente, este proceso envuelve la internalización de ideas, valores, sentimientos y comportamientos de la cultura en que está inmerso. No es de sorprender - sostiene Quiñones Rosado- que el proceso de socialización en una cultura de opresión redunde para los sectores dominados en una autoimagen negativa, en un limitado autoconcepto, en baja autoestima y un pobre amor propio. En cambio, para los miembros del grupo dominante el proceso de socialización resulta en uno positivo. Positiva autoimagen, concepto propio exagerado, una autoestima inflada y narcicismo... Particularmente hoy día, con los medios de comunicación se perpetúa esta situación porque mientras para los sectores dominantes se proyectan ciertas imágenes e información que alimentan su ego; de los sectores dominados se proyectan imágenes e información que no necesariamente, en la mayoría de los casos, nutre la autoestima, el autoconcepto y mucho menos el amor propio.

Consciousness-in-Action (2007) de Quiñones Rosado es la base conceptual que nos permite sostener que la invisibilización-hipervisibilización mediática es un sistema de opresión en las sociedades de la información. Quiñones Rosado (2007) trae a colación la fórmula: Prejuicio + Poder = Opresión. Teniendo como base lo expuesto, proponemos la siguiente fórmula:

\section{INVISIBILIDAD E HIPERVISILIZACIÓN + PODER MEDIÁTICO}

= OPRESIÓN MEDIÁTICA

Para entender esta fórmula pensemos en el caso de los residenciales públicos y comunidades especiales de Puerto Rico. La situación de opresión sistémica, en este caso, tiene doble cara. Por un lado, el capitalismo arrancó a estos sectores de los campos con el proceso de industrialización salvaje, empujándolos al arrabal y a la pobreza extrema. Por el otro, la invisibilización-hipervisibilización les está arrancando la identidad proyectando a través de los medios de comunicación masiva una identidad que no refleja en su totalidad la identidad real, pero sí afecta de forma real la autoestima, el autoconcepto y el amor propio de las personas que son parte de estos sectores. Álvarez Curbelo (2002), en la contraportada de Bajo Asedio: Comunicación y exclusión en los residenciales públicos de San Juan, nos confronta con esta situación: 
El proyecto de modernización de Puerto Rico remontó graves déficits demográficos, económicos y educativos. Para erradicar el arrabal se instauró un ambicioso programa de vivienda pública urbana. Los caseríos, más tardes renominados como residenciales, fueron un "embellecimiento estratégico" que lejos de paliar la exclusión de los sectores populares terminó por acentuarla.

En estos tiempos de crisis en las instituciones y comportamientos modernos, la exclusión adopta nuevos rostros en los residenciales de San Juan. Los medios de comunicación se suman a los dispositivos de exclusión espectacularizando la cultura de la muerte e invisibilizando otras formas de sociabilidad en los espacios de la pobreza.

Esta investigación incursiona en el rol de los medios en la construcción de las identidades en el residencial y se pregunta por las nociones de ciudadanía, democracia y paz que manejan sus habitantes a despecho de los estereotipos y las caracterizaciones mediáticas.

Esta situación de injusticia mediática es un tipo de opresión sistémica que afecta a sectores en desventaja social y económica, por ende, se debe trabajar para erradicar esta forma de opresión en la denominada Sociedad de la Información.

\section{Agenda para liberación de la opresión mediática}

Para trabajar con la situación de invisibilización-hipervisibilización de los sectores empobrecidos en los medios de comunicación masiva, Melano (2001) parece plantearque - para este tipo de problemática de exclusión- la respuesta está en el reconocimiento de la ciudadanía. Ser ciudadano supone ser portador de derechos, reconocer titularidad de derechos y tener posibilidad de su reconocimiento. Sin embargo, en una economía de mercado, ser ciudadano está muy ligado a la posibilidad de consumo. Es decir, quien no tenga los recursos económicos, tendrá una limitación en el ejercicio de su ciudadanía. Por ejemplo, el derecho a la libertad de expresión, aunque está contenido en diversas piezas legislativas y en las constituciones, está limitado porque los grupos sociales que no cuentan con los recursos económicos para costear los altos costos de los espacios mediáticos, a nuestro juicio, tienen un derecho a la libertad de expresión muy limitado pues, a nuestro entender, la participación en los medios de comunicación representa el nivel más alto de la manifestación de la libertad de expresión.

No negamos que el reconocimiento de la ciudadanía podría ser un primer paso para trabajar con la problemática de la invisibilización-hipervisibilización, pero la ruta para lograr la visibilización positiva de los sectores marginados en los medios de comunicación masiva necesita más que un simple reconocimiento de la ciudadanía. A nuestro entender, se debe trabajar para la democratización de los medios con el objetivo de que los sectores que han sido históricamente invisibilizados-hipervisibilizados, tengan acceso equitativo a los espacios mediáticos, amparándonos en la libertad de expresión reconocida por nuestra Constitución. En este sentido, concurrimos con Dussell (2007) quien aboga por democratizar los medios de comunicación. Según este autor, cada universidad, asociación, municipio, sindicato, etnia, barrio, etc., podría tener su televisión, su radio o su prensa escrita. Establecido lo expuesto, entendemos que la agenda inmediata debe ser establecer un proyecto que busque el acceso real de los sectores empobrecidos en los espacios mediáticos.

En este punto, llegamos a una agenda similar a trazada por Carlos Montaño (2000) pues, entendemos que se hace necesaria y urgente la respuesta profesional a esta problemática de Invisibilización-Hipervisibilización de los sectores empobrecidos en los medios de difusión masiva. Nos referimos a la construcción de un proyecto ético-político que concretice la justicia, equidad e igualdad para todos y todas en los medios de comunicación masiva. Como diría Montaño estamos en una "crisis que desafía a la profesión a enfrentarla y construir respuestas colectivamente" (p. 27). Añade:

Se hace necesario la clara caracterización de un proyecto progresista, fundado en principios y valores tales como: la Libertad, la Democracia sustantiva y la Ciudadanía, los Derechos Humanos, Civiles, Políticos y Sociales, la Justicia Social, las Políticas Sociales universales, nocontributivas, de calidad y constitutivas de derecho de ciudadanía, la ampliación de la esfera pública, la eliminación de toda forma de explotación, dominación y sometimiento, como sistema de convivencia social y de desarrollo de una ciudadanía sustantiva (Montaño, 2000, p. 29).

Teniendo como modelo el trabajo de Montaño, sometemos la agenda que entendemos factible para trabajar en la minimización de la invisibilización positiva e hipervisibilización negativa de los sectores empobrecidos en Puerto Rico: 
- Concienciación de los invisibilizados/as Educación de los invisibilizados/as Organización de los invisibilizados/as Acción de los invisibilizados/as para la articulación de las fuerzas vivas de la sociedad en pro de una verdadera libertad de expresión que redunde en participación en los medios de comunicación masiva.

Esta agenda debe ser trabajada de forma integral, como lo recomienda Quiñones Rosado (2007). Se trata de lo que denomina como una "práctica integral para un cambio integral" (p. 129). Hay que trabajar con los sectores invisibilizados e hipervisibilizados para que perciban, reconozcan, entiendan, y respondan de forma efectiva para lograr la liberación de la opresión mediática. Quiñones Rosado (2007) y Barreto Cortez (2000) explican este modelo integral:

- Una visión de lo que es bienestar y desarrollo integral individual y colectivo.

- Una guía para facilitar el análisis crítico de las dinámicas y circunstancias -en nosotros y en nuestra sociedad - que limitan nuestro bienestar y desarrollo integral.

- Una guía para facilitar y dirigir procesos de sanación y transformación individual y colectiva (p. 2).

Es decir, este modelo es uno creativo y dinámico de intervención que, a su vez, es una herramienta de análisis y evaluación; guía para un proceso de sanación y transformación individual y colectiva; y una visión integrada del bienestar y el desarrollo de los seres humanos. Para poder trabajar en esta dirección, se necesitan seguir ejemplos que han dejado huellas que dirigen hacia la liberación. Quiñones Rosado (2007) nos presenta dos: Saint Paul's Community Baptist Church e llé, organización para la Conciencia-en-Acción.

En el caso de los invisibilizados/as, entendemos que, al igual que las mujeres, los/as negros/as y los/as trabajadores/as, tendrán que organizarse y educarse para luchar por su derecho a la verdadera libertad de expresión, teniendo como punto de partida la conciencia-en-acción.

\section{Conclusiones}

El breve repaso de los trabajos de Quiñones Rosado (2007), Quijano (2000), Dussel (2007), Hardiman y Jackson (1997), Bronfenbrenner (1987) y Melano (2001), entre otros, puede hacernos ver cuán importante es establecer una relación saludable y positiva entre los diferentes componentes de la sociedad puertorriqueña. Concurrimos con Quiñones Rosado cuando establece que existe una coexistencia entre las dimensiones individual y colectiva. Entonces, se puede establecer que la representación negativa que constantemente proyectan los medios de comunicación masiva sobre los residenciales públicos y las comunidades especiales en Puerto Rico - por ejemplo - puede aportar a maximizar el discrimen, la marginación, el estereotipo, la baja autoestima, la inferioridad interiorizada, el rechazo y la laceración de la identidad de los sectores invisibilizados e hipervisibilizados.

De otra parte, entendemos que los medios de comunicación masiva, mediante la invisibilización de las noticias positivas e hipervisibilización de los eventos negativos que acontecen en esos sectores, están aportando a la estigmatización de los residenciales públicos y las comunidades especiales del país. El Dr. Varas Díaz (2011) menciona:

El concepto de estigma data de la antigua Grecia donde se utilizaba para describir una marca corpórea o tatuaje en la piel de una persona que evidenciaba su participación en un acto negativo y le identificaba como alguien a ser evitado/a. A la persona estigmatizada se le considera casi inhumana (p. 15).

Asimismo, explica que "el estigma no es un fenómeno únicamente cognoscitivo o de interacción social, sino un esquema y herramienta macrosocial para ejercer control y poder" $\quad$ (p. 19). A nuestro entender, los medios de comunicación masiva de Puerto Rico están perpetuando la estigmatización que se ha gestado en torno a las personas empobrecidas del país, acción que converge con las estrategias de dominación y sometimiento de la clase dominante. Con la hipervisibilización de los eventos negativos e invisibilización de lo positivo de estos segmentos poblacionales, se está identificando a los sectores empobrecidos con una "marca" que los clasifica como personas a ser evitadas. Definitivamente, este tema de la estigmatización de estos sectores debe ser estudiado y analizado en profundidad por las repercusiones sociales a nivel individual y colectivo que trae consigo esta problemática. 
Los/as profesionales de Trabajo Social del siglo XXI deben reconocer que están insertos en la era de la información y deben incluir en sus agendas la misión de: dar visibilidad a los invisibilizados.

\section{Referencias}

Anderson, Ralph (1994). La Conducta Humana en el Medio Social. Enfoque Sistémico de la Sociedad. Edit. Gedisa, Barcelona, España, Capítulos 2, 3 y 8.

Barreto Cortez, Esterla, \& Quiñones Rosado, Raúl (2007). El modelo de bienestar y desarrollo integral y sus implicaciones para la práctica del profesional de ayuda. En revista Análisis, Escuela Graduada de Trabajo Social Beatriz Lasalle, Universidad de Puerto Rico, Recinto de Río Piedras.

Bronfenbrenner, Urie. (1979). La ecología del desarrollo humano: Experimentos en entornos naturales y diseñados. Barcelona, España: Paidos.

Dussel, Enrique. (2007). Política de la Liberación, Volumen I: Historia Mundial y Crítica, Vol. 1. Madrid: Editorial Trotta.

Dussel, Enrique. (2007). Política de la Liberación, Volumen II: Arquitectónica, Vol. 1. Madrid: Editorial Trotta.

EN.FA.SIS. (2001). Tendiendo puentes: Estudio para fortalecer la relación entre los medios de comunicación y las comunidades en Puerto Rico. Proyecto de Comunicación TUTV-Canal 6 de Puerto Rico.

Greene, Roberta Rubin. (1991). The Ecological Perspective: An Eclectic Theoretical Framework for

Social Work Practice. In Greene, R.R. \& Ephross, P.H. (Eds). Human Behavior Theory and Social Work Practice. New York: Aldine de Gruyter, pp. 227-260.

Hardiman, Rita, \& Jackson, Bailey W. (1997). Conceptual Foundation for Social Justice Courses. In M. Adams Lee Anne Bell and Patt Giffin (ed.), Teaching for Diversity and Social Justice. New York: Routledge.

Hull, G. H., Mather, J. H. (2006). Understanding generalist practice with families. Belmont, CA: Thomson Brooks/Cole, 26-50.

Lander, Enrique (Compilador) (2000). La colonialidad del saber: Eurocentrismo y Ciencias Sociales. Perspectivas
Latinoamericanas. Buenos Aires: Consejo Latinoamericano de Ciencias Sociales (CLACSO).

Melano, María Cristina (2001). Ciudadanía y trabajo social: Temas para la agenda de una nueva construcción. En Un trabajo social para los nuevos tiempos: La construcción de la ciudadanía (pp. 173205). Buenos Aires, Argentina: Lumen Humanitas.

Montaño, Carlos. (2003). Hacia la construcción del proyecto ético-político profesional crítico en Borgianni y otros (Ed.) Servicio Social Crítico: Hacia la construcción del nuevo proyecto ético-político profesional. Brazil: Editorial Cortez.

Mujica, Héctor, \& Díaz Ranger, Eleazar. (1969). Los medios empresariales y libertad de prensa.

Universidad Central de Venezuela, Facultad de Humanidades y Educación, Escuela de Periodismo, Caracas.

Navedo Román, Yalitza (19 de enero de 2013). Entrevista a líder comunitario de Los Filtros en torno a los medios de comunicación y los sectores empobrecidos. San Juan, Puerto Rico.

Noelle-Neumann, Elisabeth. (1984). The Spiral of Silence. Chicago: University of Chicago.

Quijano, Anibal (1977). Imperialismo y marginalidad en América Latina. Lima: Moca Azul Editores.

Quiñones Rosado, Raúl (2007). Consciousnessin-Action: Toward an Integral Psychology of Liberation \& Transformation. Caguas: Ediciones llé.

Reguillo, Rosanna. (2000). Un mapa de silencios. En Diálogos de la comunicación, (pp. 59$60,76-86)$.

Sepúlveda, Héctor. L., García de Jesús, Yomarie, Escalante Rengifo, Guadalupe, Toro Adorno, Yolanda. (2002). Bajo asedio: Comunicación y exclusión en Residenciales Públicos de San Juan. Editorial Tal Cual: Puerto Rico. 
Stephens, Mitchell (1988). A History of News From the Drum to the Satellite. New York, USA: Viking.

Valle-Ferrer, Diana (1991). Violencia en la familia: Manifestaciones y prevención desde una perspectiva sistémica. Revista Punto y Coma III, 59-68. 